\title{
Predicting Individual Differences in Placebo Analgesia: Contributions of Brain Activity during Anticipation and Pain Experience
}

\author{
Tor D. Wager, ${ }^{1}$ Lauren Y. Atlas, ${ }^{2}$ Lauren A. Leotti, ${ }^{3}$ and James K. Rilling ${ }^{4}$ \\ ${ }^{1}$ Department of Psychology, University of Colorado, Boulder, Colorado 80309, ${ }^{2}$ Department of Psychology, Columbia University, New York, New York \\ 10027, ${ }^{3}$ Department of Psychology, Rutgers University, Newark, New Jersey 07102, and ${ }^{4}$ Department of Psychiatry and Behavioral Sciences, Emory \\ University, Atlanta, Georgia 30322
}

Recent studies have identified brain correlates of placebo analgesia, but none have assessed how accurately patterns of brain activity can predict individual differences in placebo responses. We reanalyzed data from two fMRI studies of placebo analgesia $(N=47)$, using patterns of fMRI activity during the anticipation and experience of pain to predict new subjects' scores on placebo analgesia and placebo-induced changes in pain processing. We used a cross-validated regression procedure, LASSO-PCR, which provided both unbiased estimates of predictive accuracy and interpretable maps of which regions are most important for prediction. Increased anticipatory activity in a frontoparietal network and decreases in a posterior insular/temporal network predicted placebo analgesia. Patterns of anticipatory activity across the cortex predicted a moderate amount of variance in the placebo response $(\sim 12 \%$ overall, $\sim 40 \%$ for study 2 alone), which is substantial considering the multiple likely contributing factors. The most predictive regions were those associated with emotional appraisal, rather than cognitive control or pain processing. During pain, decreases in limbic and paralimbic regions most strongly predicted placebo analgesia. Responses within canonical pain-processing regions explained significant variance in placebo analgesia, but the pattern of effects was inconsistent with widespread decreases in nociceptive processing. Together, the findings suggest that engagement of emotional appraisal circuits drives individual variation in placebo analgesia, rather than early suppression of nociceptive processing. This approach provides a framework that will allow prediction accuracy to increase as new studies provide more precise information for future predictive models.

\section{Introduction}

Though placebo treatments by definition have no direct pharmacological or physical effects, placebo administration engages brain circuits that can confer therapeutic benefits. For example, placebo painkillers reduce both pain and noxious stimulusevoked brain responses (Wager et al., 2004; Price et al., 2007; Watson et al., 2007; Colloca et al., 2008; Eippert et al., 2009a; Lu et al., 2010; Morton et al., 2010).

Placebo responses are notoriously variable across individuals (Beecher, 1955; Hoffman et al., 2005), making it difficult to eliminate them in clinical trials and harness them in treatment. Relationships between placebo effects and personality measures have proven inconsistent (Liberman, 1964; Shapiro et al., 1979), but new studies suggest a number of correlates of placebo response magnitude, in-

Received July 1, 2010; revised Sept. 15, 2010; accepted Sept. 22, 2010.

This paper was made possible with the support of National Institutes of Health (NIH) Grants MH076136 and 1RC1DA028608 (T.D.W.), as well as support from the Mind Brain Body and Health Initiative, funded by the NIH, the John D. and Catherine T. MacArthur Foundation, the Rockefeller Family and Associates, and the Kohlberg Foundation. We thank Ed Smith, Jonathan Cohen, Bob Rose, Martin Lindquist, Richie Davidson, Steve Kosslyn, Niall Bolger, Russ Poldrack, David Madigan, and Scott Schafer for helpful discussions and support on various aspects of the study design and analysis.

Correspondence should be addressed to Tor D. Wager, Department of Psychology, University of Colorado, Muenzinger D244, 345 UCB, Boulder, C0 80309-0345. E-mail: Tor.Wager@Colorado.edu.

DOI:10.1523/JNEUROSCI.3420-10.2011

Copyright $\odot 2011$ the authors $\quad 0270-6474 / 11 / 310439-14 \$ 15.00 / 0$ cluding suggestibility (De Pascalis et al., 2002; Morton et al., 2010), optimism (Morton et al., 2009), expectation (Vase et al., 2003; Zubieta et al., 2005; Atlas et al., 2010; Morton et al., 2010), behavioral activation (Schweinhardt et al., 2009), desire for relief (Vase et al., 2003), and opiate sensitivity (Amanzio and Benedetti, 1999). Brain-based correlates include individual differences in opioid system activation (Zubieta et al., 2005, 2006; Wager et al., 2007b), anticipatory activity (Wager et al., 2004, 2007b), striatal "reward" responses (Scott et al., 2007) and gray-matter density (Schweinhardt et al., 2009).

Though promising, these studies are limited by several factors. First, sample sizes have been necessarily small, limiting the reliability of individual difference measures. Second, neuroimaging studies have been designed to test for nonzero correlations between placebo analgesia and brain measures, but not to estimate predictive accuracy (effect size). A recent series of papers highlights the importance of this issue: Selecting significant voxels from maps of correlations provides inflated estimates of effect size (Vul et al., 2009; Kriegeskorte et al., 2010; cf. Lieberman et al., 2009). Smaller sample sizes and more stringent correction for multiple comparisons exacerbate the inflation (Yarkoni, 2009). Thus, a study may report correlates with placebo analgesia in many brain regions, but be useless for predicting placebo analgesia in new individuals. Multivoxel pattern-based approaches can circumvent these issues, and are increasingly used for brain-based 
prediction and classification (Haxby et al., 2001; Mitchell et al., 2008; Grosenick et al., 2009).

We reanalyze data from two published studies (Wager et al., 2004) using a patternbased regression technique, LASSO-principal components regression (LASSO-PCR), designed to both estimate the predictive accuracy of whole-brain patterns and provide interpretable maps of which voxels reliably contribute to prediction. We relate individual differences in placebo analgesia to both anticipatory activity, which reflects expectation without nociceptive input and has important influences on nociception (Porro et al., 2002; Koyama et al., 2005; Lorenz et al., 2005; Berns et al., 2006; Keltner et al., 2006; Brown et al., 2008a,b; Watson et al., 2009; Atlas et al., 2010; Ploner et al., 2010), and pain processing. In addition, to provide insight into the nature of anticipatory responses, we compared the predictive power of anticipatory activity in functional networks associated with cognitive control, emotional appraisal, and pain.

\section{Materials and Methods}

\section{Summary of analyses}

A schematic summarizing the analyses we conducted is shown in Figure 1A. Analyses 1-3 examined the relationship between placebo analgesia and brain activity during pain anticipation. We first report standard voxelwise analyses of anticipatory predictors of placebo analgesia (analysis 1). This analysis uses a standard regression approach that is appropriate for inference, but provides biased estimates of effect sizes (Vul et al., 2009; Yarkoni, 2009; Kriegeskorte et al., 2010). Supplementary analyses group significant regions into functional networks (see supplemental Figs. 1-3, available at www.jneurosci.org as supplemental material). In analysis 2, we use a cross-validated, penalized regression procedure, LASSO-PCR (see Regression techniques) (see Fig. 1; supplemental Fig. 3, available at www. jneurosci.org as supplemental material) to assess how accurately anticipatory activity can predict placebo analgesia in an unbiased fashion. In analysis 3 , we assessed the contributions of anticipatory activity within several previously identified brain systems thought to be critical for placebo analgesia (Benedetti et al., 2005; Vase et al., 2009). We compared the predictive accuracy of anticipatory activity within anatomical masks derived from meta-analyses of cognitive control, emotional appraisal, and pain (high vs low noxious stimulus intensity). In analyses 4 and 5, we examined the contributions of brain responses during painful stimulation. In analysis 4 , we used the average [placebo - control] contrast values in an independent pain localizer as the outcome, and used LASSO-PCR to find patterns of anticipatory activity that predicted the magnitude of placebo effects on brain activity during pain. Finally, in analysis 5, we tested whether changes in pain-related activity are predictive of analgesia. Together, these analyses provide insight into (1) how anticipatory brain processes are related to placebo analgesia; (2) how anticipatory brain processes are related to placebo effects on brain activity during pain experience; and (3) how brain activity during pain experience is related to placebo analgesia.

\section{Participants}

De-identified data from 47 participants were included in the reanalysis. Twenty-four individuals from Princeton University participated in study
1 , and 23 individuals from the University of Michigan participated in study 2 (Wager et al., 2004). Participants gave voluntary consent in accord with the Declaration of Helsinki, and studies were approved by Princeton University and University of Michigan institutional review boards.

\section{Procedure}

Full details for procedures used in study 1 and study 2 have been published previously (Wager et al., 2004). Briefly, study 1 participants underwent fMRI while experiencing painful and nonpainful shocks on the right wrist. Subjects were informed that the application of an "analgesic" skin cream would reduce the pain intensity (placebo condition), whereas application of a "control" cream would not have any effect on pain intensity (control condition). The same inert substance was applied in each case. The order of application (analgesic vs control cream) was counterbalanced across subjects. On each trial, a visual cue indicated whether the upcoming stimulation would be painful or mild, and the subsequent anticipation period varied between 3 and $12 \mathrm{~s}$. Following a $6 \mathrm{~s}$ stimulation period, participants rated overall pain intensity on a visual analog scale from 1 to 10 .

Study 2 participants underwent fMRI while experiencing painful heat on the left forearm. Thermal heat was applied to a skin site with the 
"analgesic" cream and to a skin site with the "control" cream in different blocks. Block order was counterbalanced across subjects. At the start of each trial, the cue, "Get Ready," was presented on the screen for $1 \mathrm{~s}$, followed by a jittered anticipation interval varying from 1 to $16 \mathrm{~s}$ $($ mean $=9.77 \mathrm{~s})$. Thermal stimulation was applied for $20 \mathrm{~s}(17 \mathrm{~s}$ at peak, $1.5 \mathrm{~s}$ ramp up and down), and after a brief jittered delay, participants rated overall pain intensity on a 10-point numerically anchored visual analog scale (VAS), similar to scales commonly used in clinical practice. Fractional values, e.g., 7.72, were recorded on the computerized VAS and analyzed.

The type of pain rating scale we used has the advantages of simplicity and the ability to obtain reliable, repeated measurements for a wide range of individuals (Chapman et al., 1985; Bijur et al., 2001) in a few seconds during scanning. However, an important drawback is that use of these scales assume that pain is a unidimensional experience characterized by intensity (Bijur et al., 2001). Multidimensional inventories have shown that pain can have a number of different qualities (Clark et al., 2002), the most prominent of which have been sensory/discriminative and affective/unpleasantness aspects (Melzack and Casey, 1968; Price et al., 1987). There is much evidence that the sensory and affective aspects of pain are dissociable under some circumstances (Gracely et al., 1979; Price et al., 1980, 1987); however, pain intensity and affect ratings are highly correlated in many settings [e.g., $r=0.96$ in a study by Coghill et al. (1999)]. There is also evidence that participants make VAS affect judgments based on intensity, even when instructed otherwise, unless verbal anchors obtained using cross-modality matching are used (e.g., Gracely et al., 1979), and that pain intensity on a unidimensional scale is more closely related to its emotional qualities than its sensory ones (Clark et al., 2002). We considered measuring pain unpleasantness and intensity in pilot testing for study 2 , but intensity and affect ratings were correlated $>0.9$, consistent with common findings in the literature. Thus, we chose to use a single rating scale based on intensity. Thus, placebo analgesia scores in this study may be driven by changes in either the intensity or unpleasantness of pain, or both components.

\section{Data analysis}

All images underwent standard preprocessing (for details, see Wager et al., 2004) and BOLD activity was estimated for each brain voxel within each subject using a general linear model. For study 1 , BOLD activity was modeled for the anticipation epoch and during stimulation (pain), yielding fMRI activation estimates for each period. For study 2, BOLD activity was modeled separately for the trial onset cue, the anticipation period (which varied in duration and was modeled with a variable-duration epoch), early pain ( $0-10 \mathrm{~s})$, peak pain (10-20 s), and late pain (10 s after pain offset). All preprocessing and individual-subject level analyses were identical to those reported in the original study (Wager et al., 2004). Unless otherwise noted, we report results significant at $p<0.001$ (onetailed, uncorrected) with a cluster extent threshold of three voxels. $p<$ $0.001(Z>3.1)$ is the most common significance threshold in neuroimaging studies (Wager et al., 2007a). While this threshold does not ensure a $<0.05$ chance of finding any false positives, it provides a balance between power and false positives more conducive to replication than the use of very high thresholds.

Placebo analgesia was defined as the difference in average reported pain for the placebo $(\mathrm{P})$ condition relative to the control (C) condition for each participant. A successful placebo manipulation should result in lower pain reports for the placebo condition. As a result, larger differences in pain reports for the $[\mathrm{C}-\mathrm{P}]$ contrast (and higher resultant placebo analgesia scores) reflect stronger placebo effects on pain experience. Brain measures consisted of placebo effects on fMRI activity [P vs $\mathrm{C}$ ] during the anticipation period (Antic) and during pain administration (pain) for each study. In study 1, only one pain period was modeled: $6 \mathrm{~s}$ pain stimulation period. In study 2 , painful stimuli were applied over a period of $20 \mathrm{~s}$, resulting in three separately modeled epochs (early, peak, and late pain). The peak pain regressor from study 2 was used for the combined analysis presented here.

In this paper, we focus specifically on placebo effects in anticipation and on responses to noxious stimulation. Our previous paper published with these data specifically assessed the effects of placebo on noxious versus non-noxious stimuli and found that placebo responses were larger for painful stimuli, and were nonsignificant for innocuous stimuli (Wager et al., 2004); this finding was replicated in a later study (Wager et al., 2007b). In our previous work, we have found that there is little placebo effect during nonpainful warmth in either pain reports or pain-evoked brain responses. For this reason, many published papers focus on placebo responses during painful stimulation alone (Lieberman et al., 2004; Price et al., 2007; Craggs et al., 2008; Eippert et al., 2009a; Schweinhardt et al., 2009; Watson et al., 2009). Therefore, we felt it most appropriate here to focus on the comparison of most theoretical interest (and for which we have complete data for both experiments), placebo effects during painful stimulation. However, not all authors who have tested graded painful stimuli have found placebo effects specific to pain (Kong et al., 2006), and anticipatory responses may well be part of a more general mechanism that does not only affect pain. These considerations motivated our focus on responses to noxious stimulation in the present paper.

Because the two studies involved different procedures, pain stimuli (shock vs thermal), and fMRI scanners, it cannot be assumed that the pain intensity ratings or measured fMRI BOLD signals were similarly scaled across the two studies. To collapse across study samples, we used a nonparametric analysis that does not make strong assumptions about scaling or the normality of the data. Within each study, fMRI BOLD data from the $[\mathrm{C}-\mathrm{P}]$ Antic and $[\mathrm{C}-\mathrm{P}]$ pain contrast maps were rankordered across participants. Ranking allowed us to conduct a multiple regression equivalent of a nonparametric analysis, i.e., Spearman's $\rho$ or a Kruskal-Wallis ANOVA, which operate on rank data. For each brain voxel, the contrast values were replaced with their within-study rank (i.e., 1-24 for study 1, and 1-23 for study 2), generating a set of rank images for each contrast in each study. Rank images were combined across the two studies, and $[\mathrm{C}-\mathrm{P}]$ Antic and $[\mathrm{C}-\mathrm{P}]$ pain contrasts were analyzed separately. Behavioral data were likewise rank-ordered within each study, such that the subject in study 1 with the highest placebo analgesia score was given a ranking of 1 and the subject with the lowest score was given a ranking of 24 . The ranked data were used for all analyses.

\section{Regression techniques}

We used two kinds of regression techniques: standard robust multiple regression ("Standard regression") and LASSO-PCR, described below. For both techniques, in addition to placebo analgesia, the following covariates were also included in all regression analyses: a study indicator variable ("study," study 1 vs study 2) and the treatment administration order ("order," placebo or control first). For standard multiple regression analyses, an order by placebo analgesia interaction term was also included. The categorical variables (study and order) were contrast coded (with values of 1 or -1 ), and the placebo analgesia variable was centered before calculating the interaction regressor.

Standard multiple regression. Robust general linear model estimation was performed using iteratively reweighted least squares (IRLS) in Matlab software (MathWorks), which yields valid $p$ values while minimizing the influences of outliers and violations of normality (Wager et al., 2005). This process is shown graphically in Figure $1 B$.

Network analysis. We conducted a cluster analysis on the suprathreshold regions from the standard regression analysis (Wager et al., 2007b, 2008) to provide information about the structure of interrelationships among regions (i.e., functional connectivity). This analysis also provided information on whether the voxels correlated with placebo analgesia contain redundant information, or whether several separable networks independently predict responses. If the former were true, then patternbased predictive analyses would likely offer little advantage over using single regions. If, however, there are multiple networks that independently predict placebo analgesia, then a pattern-based approach would be advantageous because it can identify combinations of voxels/regions that are more predictive than any single one alone. The cluster solution revealed a best-guess estimate of five networks of intercorrelated regions (see supplemental Fig. S1, available at www.jneurosci.org as supplemental material). 
LASSO-PCR. The standard regression approach described above can identify regions that are correlated with placebo analgesia across studies, but each voxel is treated independently, ignoring the influence of other voxels. In the standard approach, placebo analgesia is the predictor, and brain data from one voxel is the outcome. A different approach is needed to quantify how much of the variance in placebo analgesia can be predicted by anticipatory activity across sets of voxels. In the LASSO-PCR technique, placebo analgesia is the outcome, and a set of voxels constitutes a set of predictors.

The LASSO-PCR technique was designed to overcome three obstacles to providing unbiased estimates of predictive accuracy across large numbers of voxels. First, standard regression slopes (weights) are highly variable, particularly with many predictors, reducing prediction accuracy as the number of predictors increases. Second, predictors fit noise as well as true effects, so a good fit to a particular dataset does not guarantee that the model will predict accurately for new observations. A popular solution is cross-validation (Shao, 1993; Kohavi, 1995; Hastie et al., 2005), the division of a dataset into two mutually exclusive partitions: a training set, on which the regression model is fit, and a test set, on which the model's predictions are evaluated. Finally, with many voxels, multicollinearity among the predictors (voxels) will render standard regression slopes uninterpretable. The regression slopes are weights on voxels that constitute a map of the direction and magnitude of contributions to the prediction-thus, the multicollinearity issue must be addressed before the regression procedure can yield maps of the contribution of each voxel to the prediction that are neuroscientifically interpretable. Modern regression techniques used in machine learning can provide solutions to both of these problems (Michalski et al., 1986; Bishop, 2006; Genkin et al., 2007). Regularization techniques such as ridge regression and, more recently, LASSO (least absolute shrinkage and selection operator) (Tibshirani, 1996), shrink the regression weights toward zero, but in so doing can stabilize the regression weights (i.e., reduce their variance) and provide a principled way of selecting a subset of predictors.

The LASSO-PCR procedure, illustrated in Figure $1 C$, is a combination of several established techniques chosen to work well for fMRI data. Predictors consist of a (usually large) set of brain voxels (e.g., [P $-\mathrm{C}]$ contrast values for anticipatory activity within cortical regions), and the outcome is a single variable (e.g., placebo analgesia). Voxels in fMRI data are highly correlated because they are grouped into networks. LASSO regression would ignore that covariance and tend to select a few voxels, which would reduce interpretability. To avoid this issue, LASSO-PCR uses principal components analysis (PCA) to reduce the dimensionality of the data first, and then uses LASSO regression on the component scores. LASSO regularization provides a principled way of selecting a subset of distributed components and weights on each that together best explain the training data. LASSO has favorable properties compared with ridge regression or best-subsets selection of predictors (Hastie et al., 2005). Unlike ridge regression, it tends to select a subset of predictors, simplifying the regression model and enhancing interpretability (Hastie et al., 2005). Unlike best-subsets selection, it provides a smooth constraint on inclusion of each predictor in the regression model.

For the PCA step, we retained a full-rank set of components $(45 \mathrm{com}$ ponents). For LASSO regression of outcomes ( $\mathbf{Y})$ on component scores (S), we used the least angle regression algorithm (Efron et al., 2004) as implemented in Matlab by Guilherme Rocha and Peng Zhao (http:// www.stat.berkeley.edu/ gvrocha/gvrocha_software.html). This amounted to standard regression with component scores as predictors, i.e., $\mathbf{Y}=\mathbf{S} \beta$ $+\varepsilon$, with $\mathbf{S}=\mathbf{X V}$ for original data matrix $\mathbf{X}$ and eigenvector matrix $\mathbf{V}$, subject to an additional constraint on the L1-norm of $\beta$, i.e., $\Sigma_{j}\left|\beta_{j}\right| \leq c$, where $c$ is a constant. This is accomplished by imposing a soft penalty on the solution governed by a weight parameter, $\lambda$. In practice, a minimal penalty was used $\left(\lambda<3 \times 10^{-5}\right)$, so that results were close to the standard PCA regression solution. For each analysis reported, we assessed accuracy with the covariates alone (study 1 vs 2 and order of placebo administration), the brain alone, and the combined brain and covariates. The covariates never appreciably reduced the prediction error in any analyses (they were never significantly correlated with the outcome) and are not discussed further. Results from the brain data plus covariates analyses are reported, though they were virtually identical to the brain-data-only analyses in all cases.
Cross-validation. Cross-validation provides a minimally biased estimate of predictive accuracy, even with complex or "overfit" models (in this case, regression with many predictors). The data are divided into training and test sets of independent observations (here, subjects). The model is developed and all parameter estimates are chosen on the training data, and predictions are made for the test data. The training-test split and model fitting are repeated so that each observation is used as a test observation exactly once. This way, each observation is used for both model selection and accuracy assessment, but because the training and test data are separated and independent, even complex models cannot "capitalize on chance," and the procedure provides a minimally biased estimate of the accuracy for predicting responses in new individuals. We used a stratified fourfold cross-validation procedure (Kohavi, 1995), so that the regression model was always fit on data from $3 / 4$ of the subjects and tested on the remaining $1 / 4$. The analysis was repeated four times (folds), each time leaving out a different subset of subjects. The test sets were selected so that the set in each fold had approximately the same distribution on the outcome variables. This procedure is known to underestimate the predictive accuracy (i.e., there is a small bias toward zero), but provides less variable results than the popular leave-one-out cross-validation scheme (Kohavi, 1995; Zeng and Martinez, 2000).

Prediction accuracy assessment. We assessed accuracy in two ways: first, by correlating predicted scores with observed outcome (e.g., placebo analgesia scores), which provides an intuitive plot of the relationship, and second, by assessing the PE (the mean squared deviation between predicted and standardized observed scores). A PE of 1 indicates no reduction in variance explained by the combined set of brain voxels. PE scores above 1 indicate an increase in variance, i.e., including the brain predictors is worse than not including them. This is possible because using noisy predictors will increase the noise variance in the predicted outcomes. PE scores below one indicate that the predictors are useful. We compared $\mathrm{PE}$ with brain predictors $\left(\mathrm{PE}_{\mathrm{brain}}\right)$ against $\mathrm{PE}$ under a "null model" in which only the mean outcome score for the training dataset was assumed to be known $\left(\mathrm{PE}_{\text {null }}\right)$. (In the null model, the predicted outcome for each test observation was simply the mean of the outcome scores for the training data.) We calculated variance explained by the brain patterns as $1-\left(\mathrm{PE}_{\text {brain }} / \mathrm{PE}_{\text {null }}\right)$.

Significance test for prediction and bias assessment. To test the overall significance of the model and ensure that cross-validated prediction errors were unbiased, we conducted a permutation test on the LASSO-PCR procedure. For each of 1000 iterations, we permuted the outcome variable and then repeated the cross-validated LASSO-PCR procedure. If the prediction accuracy is unbiased, correlations between the predicted outcome and actual outcome values for permuted data should be, on average, zero. These correlations were indeed near zero $(r= \pm 0.02)$ for all analyses, as expected, providing a validity check and ensuring that estimates of prediction accuracy are unbiased. $p$ values for the accuracy were obtained by comparing the PE for the correct permutation with the distribution of PE over the random permutations.

Localization of predictive voxels. The regression weights from the LASSO-PCR procedure are projected back into voxel space $(W=V \beta)$, where $W$ is a vector of voxel weights that is then reconstructed into a 3-D image, and thus constitute a map of the magnitude and direction of each voxel's contribution to the prediction. To assess which voxels make reliable contributions, we performed a bootstrap test (bias-corrected and accelerated; Efron and Tibshirani, 1993) taking 1000 samples with replacement from the paired predictor and outcome data and repeating the cross-validated LASSO-PCR for each bootstrap sample. The bootstrap provides a way of assessing $p$ values based on the percentage of bootstrap samples that fall above the critical test value of 0 in each voxel (see Wager et al., 2009a for more details). Thresholding based on voxelwise $p$ values provides information about which voxels make the most reliable contributions to prediction, though it is important to note that predictions are made based on the full set of weights across all voxels.

Localization of pain-processing network from independent data Pain-processing network (PPN) regions were localized by a megaanalytic approach that assessed intensity processing across five experi- 
ments that all contrasted high- versus low-intensity noxious thermal stimulation of the left volar forearm (total $n=114$ ). All participants gave informed consent and procedures were approved by the Columbia University Institutional Review Board. Individual subjects' contrasts between high- and low-intensity painful stimulation (with temperatures chosen individually for each subject based on a standardized adaptive staircase calibration procedure) were transformed to $z$-scores across image space within each individual (to avoid scaling issues resulting from the use of different basis sets and pain epoch durations) and normalized to MNI space using SPM5's unified segmentation algorithm. The group model included an intercept and covariates coding for differences among the five studies. We included these normalized images in a one-sample $t$ test of [high - low intensity] contrast values across the three different studies. Familywise error correction $(p<$ 0.05 ) using Gaussian random fields as implemented in SPM5 was used to identify voxels showing a significant [high - low intensity] effect, which was used to define the "pain-processing network." The following anatomical regions were found to comprise the PPN: bilateral superior, inferior, and middle frontal gyrus; bilateral precentral gyrus; right middle and bilateral lateral orbitofrontal gyrus; bilateral postcentral gyrus; bilateral supramarginal gyrus; bilateral superior and middle temporal gyrus; right hippocampus; right parahippocampal gyrus; left lingual gyrus; bilateral insular cortex; bilateral cingulate gyrus; bilateral caudate; bilateral putamen; brainstem; and cerebellum, bilateral thalamus, and medial thalamus. These results are shown in supplemental Figure S4 (available at www.jneurosci.org as supplemental material).

\section{Analyses}

Analysis 1: anticipatory predictors of placebo analgesia using standard multiple regression. Across our pooled sample, we searched for patterns of activity during anticipation of pain that were predictive of the magnitude of reported placebo analgesia. We also conducted supplementary cluster analysis on the resulting regions to group them into networks and test whether multiple networks make separable contributions to placebo analgesia.

Analysis 2: anticipatory predictors of placebo analgesia using LASSO$P C R$. As discussed above, selecting voxels showing significant correlations with placebo analgesia and then reporting their effect sizes is a suitable way to test for nonzero correlations, but it produces biased estimates of effect size. LASSO-PCR is suited to providing unbiased estimates of how much of the variance in placebo analgesia can be predicted by anticipatory activity across sets of voxels. The main goal of analysis 2 was to assess the predictive accuracy for new subjects based on patterns across large areas of the brain specified a priori. A second goal was to interpret the maps of LASSO-PCR predictive voxels and compare them with the standard correlations in analysis 1 . If the maps from the pattern-based regression procedure are interpretable, the voxel weights should be reliable and some of them should overlap with those identified in analysis 1 . However, because the pattern-based analysis considers multiple brain predictors simultaneously, it is possible that it may reveal new regions that are not significant in the standard multiple regression analysis, but are important in the context of other brain predictors.

Accurate predictions depend not only on having strong predictive signals in the data, but also on being able to identify the most predictive variables a priori (i.e., there is a cost to including additional "junk" variables). Thus, accuracy would theoretically be improved if the most relevant voxels could be identified before conducting analyses. We used LASSO-PCR to make predictions about placebo analgesia scores from large areas of the cortex selected a priori from the LONI anatomical probabilistic atlas (Shattuck et al., 2008). The mask was based on results from other studies of placebo, anticipatory anxiety, and cognitively generated emotion [based on results from Benedetti et al. (2005) and newer published findings], but it made very weak assumptions about localization: The mask covered very broad areas of the cortex, including prefrontal, parietal, and cerebellar regions.

Analysis 3: comparison of predictive accuracy of anticipatory activity in regions associated with emotional appraisal, cognitive control, and pain.
LASSO-PCR provides an estimate of how well an ensemble of voxels predicts a given outcome. We took advantage of this feature and compared how accurately placebo analgesia was explained by anticipatory activity within three a priori masks (voxel ensembles). The purpose was to test whether regions associated with particular classes of tasks predicted placebo analgesia better than others, and thereby to gain some insight into the functions of the brain networks subserving placebo analgesia. We defined masks based on meta-analyses for three types of processes: emotional appraisal, executive working memory (which involves multiple cognitive control and maintenance processes), and pain. Though these systems have all been indirectly implicated in placebo- and anticipation-based modulation of pain (Benedetti et al., 2005; Koyama et al., 2005; Boly et al., 2007; Aslaksen and Flaten, 2008; Lyby et al., 2010), it is unknown whether activity in these systems is predictive of the magnitude of placebo analgesia.

The masks for emotional appraisal and working memory were taken from two previous meta-analyses: a meta-analysis of emotion involving 163 studies (Kober et al., 2008) and a meta-analysis of executive working memory versus simple storage involving 60 studies (Wager and Smith, 2003). For pain, we used the PPN localizer described above (see Localization of pain-processing network), which produced results highly consistent with other studies of noxious stimulus intensity coding (Coghill et al., 1999; Peyron et al., 2000) (see supplemental Fig. S4, available at www.jneurosci.org as supplemental material). We ran LASSO-PCR within each mask, noting as the primary outcome the variance in placebo analgesia explained by brain activity, compared with the "null model."

Analysis 4: anticipatory predictors of noxious stimulus-evoked painprocessing network responses using LASSO-PCR. In addition to placebo analgesia, which is a self-report-based measure, we related anticipatory brain activity with individual differences in $[\mathrm{C}-\mathrm{P}]$ activity during noxious stimulation. We were particularly interested in whether anticipatory activity predicted placebo effects on pain-evoked responses within regions specifically responsive to noxious stimuli.

To form a simple, robust outcome measure, we extracted pain period $[\mathrm{C}-\mathrm{P}]$ contrast values for each subject in each voxel identified in the PPN localizer contrast and averaged across voxels to obtain a single measure of placebo effects on PPN activity. We then used LASSO-PCR to identify how accurately anticipatory activity predicts placebo effects on pain-evoked responses, and to create a map of the strength of each voxel's contribution to the prediction.

Analysis 5: pain-period predictors of placebo analgesia. Finally, we used the LASSO-PCR approach described above to assess the extent to which pain-evoked responses predict placebo analgesia. We again used the PPN localizer described in analysis 3 above as a mask. We also ran the LASSOPCR regression with a whole-brain mask, and compared the resulting voxel weights with standard regression weights for the relationship between $[\mathrm{C}-\mathrm{P}]$ activity during peak pain and placebo analgesia. This final analysis allowed us to test whether pain-period activity predicted placebo responses and whether the pattern of predictive voxels is consistent with overall inhibition of the PPN in strong placebo responders or a more complex pattern of changes.

\section{Results}

\section{Anticipatory predictors of placebo analgesia (analyses 1-3)}

Standard regression analyses (analysis 1)

Across the two studies, participants who reported greater placebo analgesia $[\mathrm{C}-\mathrm{P}]$ demonstrated greater placebo-induced anticipatory activity $[\mathrm{P}-\mathrm{C}]$ in the cerebellum, inferior parietal lobule, and regions throughout the prefrontal cortex (PFC), including the dorsolateral PFC (DLPFC), inferior frontal gyri (IFG), orbital frontal cortex (OFC), anterior PFC, and supplementary motor area (SMA) (red/yellow in Fig. 2A; see supplemental Table S1, available at www.jneurosci.org as supplemental material). Each of these prefrontal regions has been shown to exhibit placeboinduced increases during anticipation in other voxelwise analyses of placebo analgesia (Lieberman et al., 2004; Wager et al., 2004; Watson et al., 2009; Lu et al., 2010). 


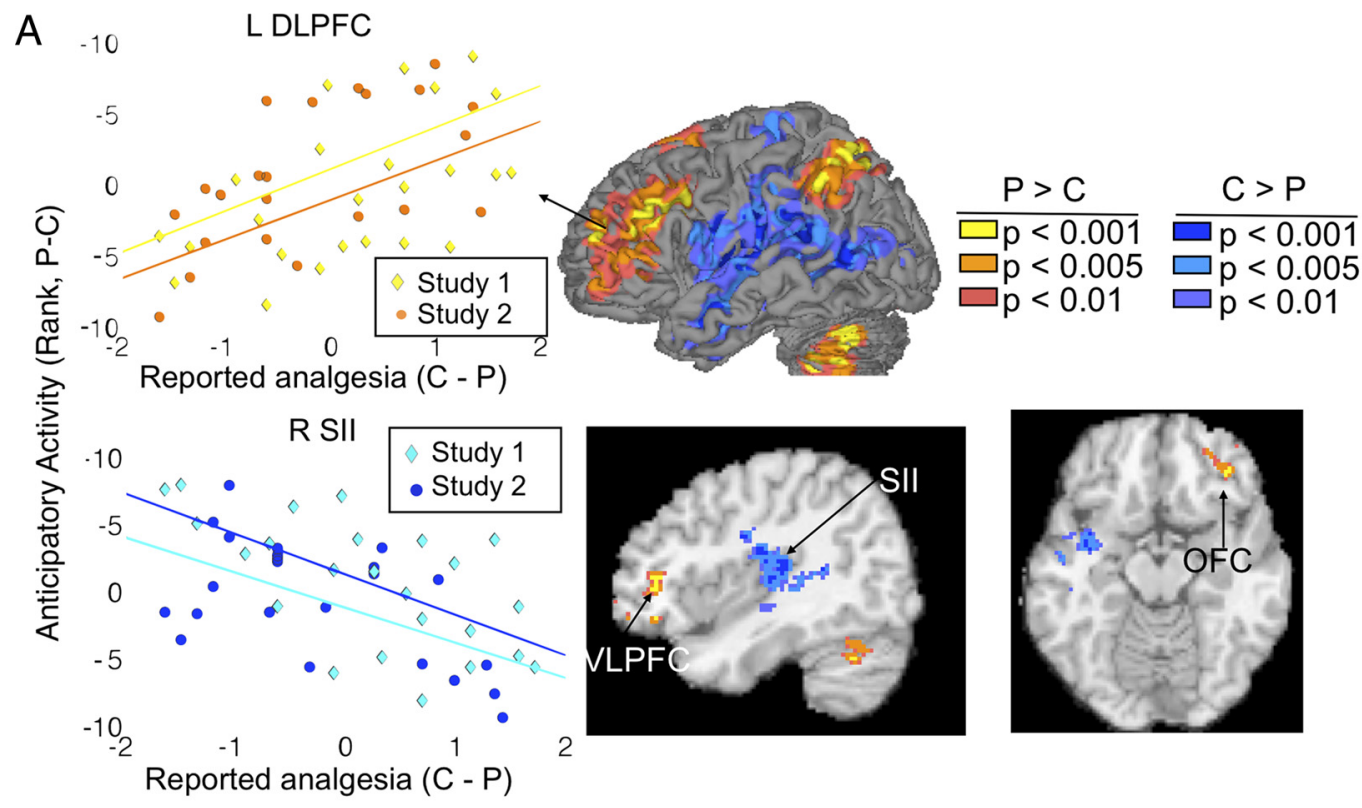

B

\section{Correlations with expected analgesia (Study 2)}
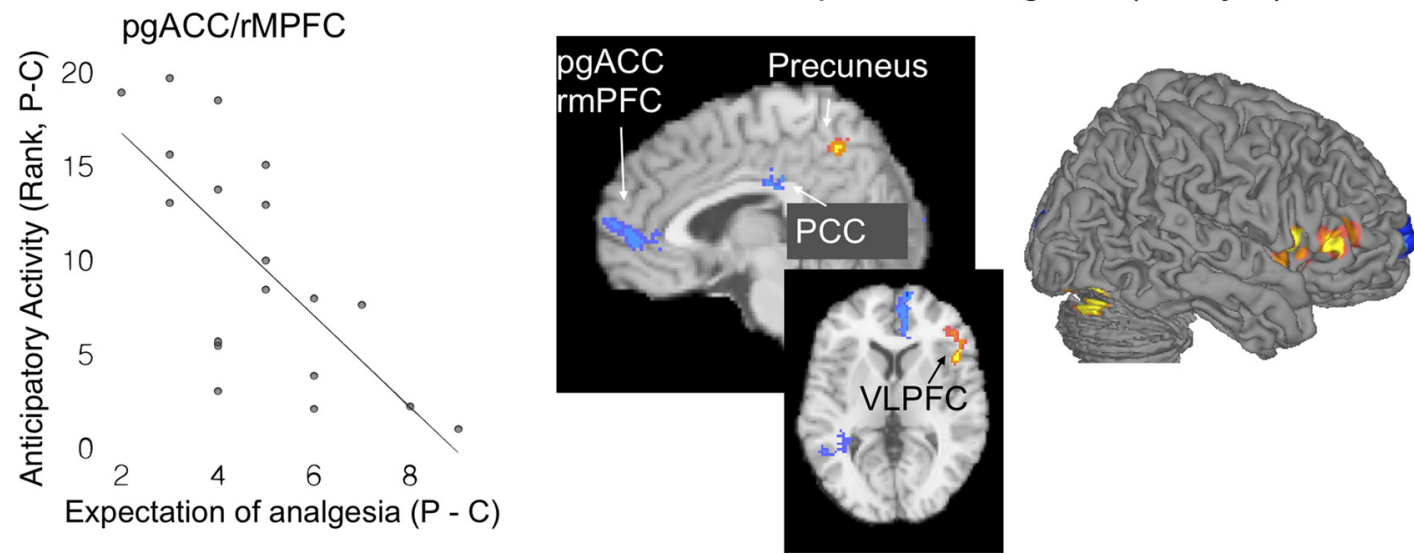

Figure 2. Anticipatory predictors of placebo analgesia from standard regression. $\boldsymbol{A}$, Surface figures (middle) illustrate regions where greater placebo related increases (warm colors) and decreases (cool colors) during pain anticipation predicted greater placebo analgesia. Scatter plots (left) illustrate the relationship between average cluster activity during anticipation ( $y$-axis) and reported placebo analgesia ( $x$-axis). Examples are shown for the left DLPFC (positive correlation) and for the right SIl region (negative correlation). BOLD activity reported on the $y$-axis ascends from top to bottom, because activity is displayed relative to placebo condition, where the contrast values were negative when $P>C$ and positive when $C>P$. The slices (right) show in greater detail that increases predicted analgesia in the VLPFC and OFC, while decreases predicted analgesia in SIl and a region encompassing the insula and superior temporal gyrus (STG). $\boldsymbol{B}$, Regions in which anticipatory activity was correlated with greater prescan expectations of analgesia in study 2. The scatter plot shows the negative correlation in pgACC/rmPFC, averaged across voxels.

Individual differences in placebo analgesia were also predicted by relative decreases during anticipation $[\mathrm{C}-\mathrm{P}]$. Greater placebo analgesia was associated with less placebo-related anticipatory activity (Fig. $2 \mathrm{~A}$, blue) in a large area of the parietal operculum and temporal cortex encompassing bilateral SII, as well as right posterior and rostral dorsal anterior cingulate cortices (rdACC)/anterior mid-cingulate (Vogt, 2005), as well as in the temporal pole and subcortically in the globus pallidus (GP). Our results show that relative placebo nonresponders show greater responses in these areas in placebo than in control conditions, whereas there is a relative suppression during the placebo condition in strong placebo responders. Thus, these findings are consistent with findings of engagement of these regions during aversive pain anticipation (Koyama et al., 2005). Surface renderings of regions showing both positive and negative associations with placebo analgesia are shown in Figure $3 A$, for comparison with LASSO-PCR. Below, in Analysis 3, we present additional results on whether these results are likely to be localized in "painprocessing" regions.

Network analysis. Cluster analysis on the correlations among significant regions (Wager et al., 2007b, 2008) revealed that they were organized into a best-guess estimate of five networks (permutation test $p<0.001$, compared with the null hypothesis of no functional grouping). Stepwise regression on these five networks yielded a solution in which three of the five networks were independent predictors of placebo analgesia, as shown in supplemental Figure S2 and supplemental Table 3 (available at www. jneurosci.org as supplemental material). Anticipatory activity in two of these networks-a frontal-parietal network (red in Fig. $3 B$ and supplemental Fig. S2, available at www.jneurosci.org as supplemental material) and an anterior frontal region that formed its own "network" (yellow)—was positively correlated with placebo analgesia (anticipatory increases predict analgesia), whereas anticipatory activity in an SII-temporal network (blue) was in- 
A Standard regression (Analysis 1)

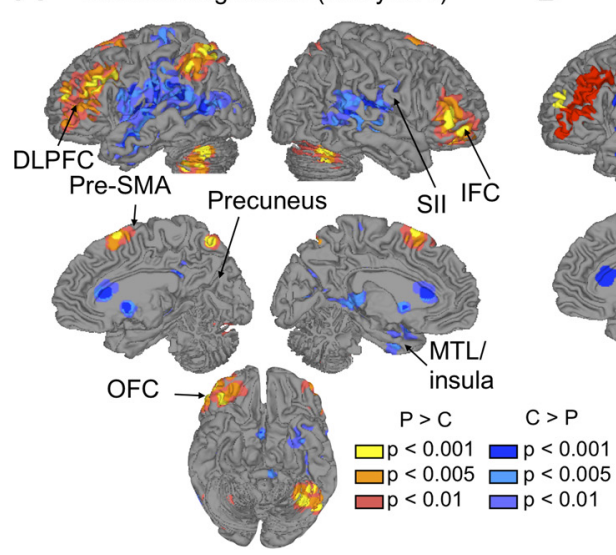

B Networks (Analysis 1)

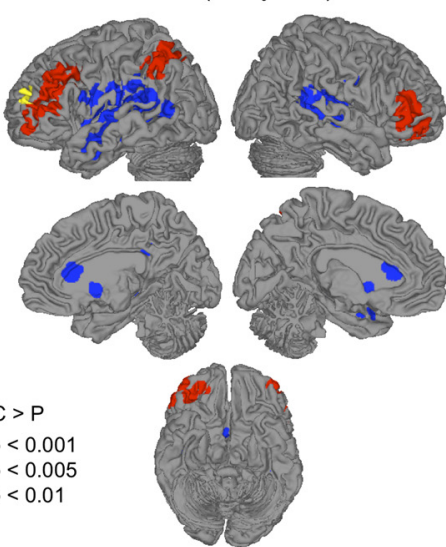

C LASSO-PCR (Analysis 2)

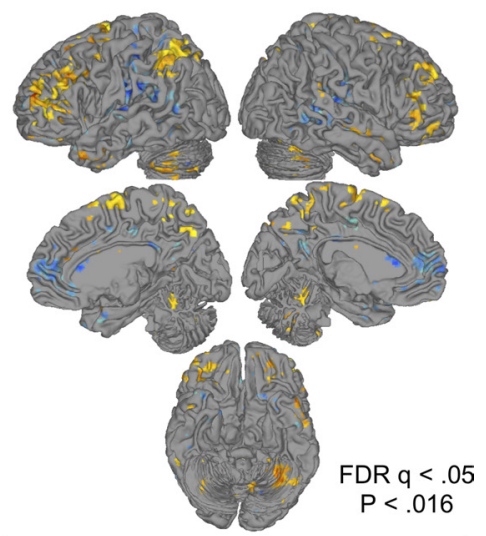

Figure 3. Anticipatory predictors of placebo analgesia, comparison across methods. $\boldsymbol{A}$, Surface figures showing results for standard regression (analysis 1 ). $\boldsymbol{B}$, Grouping of significant voxels in analysis 1 into functional networks (see supplemental material, available at www.jneurosci.org, for details). Colors indicate distinct groups of intercorrelated regions. $\boldsymbol{C}$, Weights (regression slopes) reliably predictive of placebo analgesia in the LASSO-PCR analysis (FDR $q<0.05, p<0.016$ ). Predictions for new subjects are obtained by multiplying the entire weight map (not only the colored regions) by the subject's contrast values. The most reliably predictive voxels (colored) aid in interpretation of the functional anatomy of predictive systems. Yellow/red colors indicate positive effects, greater placebo analgesia with greater placebo - control increases. Blue colors indicate negative effects, greater analgesia with reduced placebo - control activity (or placebo-related deactivation).

versely related to placebo analgesia (lower increases or deactivation associated with analgesia). More generally, these results suggest that prediction approaches that jointly consider effects in multiple brain regions may be appropriate.

Relationship with expectations. Correlations with prescan expectancy ratings in study 2 revealed correlations between $[\mathrm{P}-\mathrm{C}]$ differences in anticipatory activity and prescan expectancies of analgesia. As shown in Figure $2 B$ (and reported in supplemental Table S2, available at www.jneurosci.org as supplemental material), VMPFC and posterior cingulate showed less placebo-related $(\mathrm{P}-\mathrm{C})$ anticipatory activity with stronger expectations of analgesia (blue in Fig. $2 B$ ), whereas ventrolateral prefrontal cortex (VLPFC) and precuneus showed greater anticipatory increases with stronger expectations (orange/yellow) (cf. Lieberman et al., 2004). VLPFC correlations with expectations are consistent with the findings above on VLPFC prediction of placebo analgesia and with other published findings (Lieberman et al., 2004). Notably, however, VMPFC results did not predict analgesia in the standard regression results reported above. We return to this issue below.

Lasso-PCR regression (analysis 2)

We next used LASSO-PCR to (1) assess the predictive accuracy for new subjects based on patterns of activity across the cortex and (2) interpret the maps of predictive voxels and compare them with the standard regression results in analysis 1.

An unbiased estimate of predictive accuracy was obtained using LASSO-PCR within a broad a priori cortical mask constructed to cover general areas associated with placebo effects in other studies. The mask included frontal, temporal, and opercular regions, covering much of the cortex but excluding motor, occipital, and high parietal regions not thought to be associated with placebo effects. Prediction-outcome correlations were $r=$ 0.37 [a $12 \%$ reduction in $\mathrm{PE}, p=0.03$ ], and were different across studies, as shown in Figure $4 A$. The correlation for study 1 was $r=0.05$ (an 11\% increase in PE using brain activity), and the correlation for study 2 was $r=0.64$ (a $40 \%$ reduction in PE). A separate LASSO-PCR analysis run on study 2 alone revealed a higher correlation, $r=0.73$ (Fig. $4 B$ ) (a $54 \%$ reduction in $\mathrm{PE}$ ), suggesting
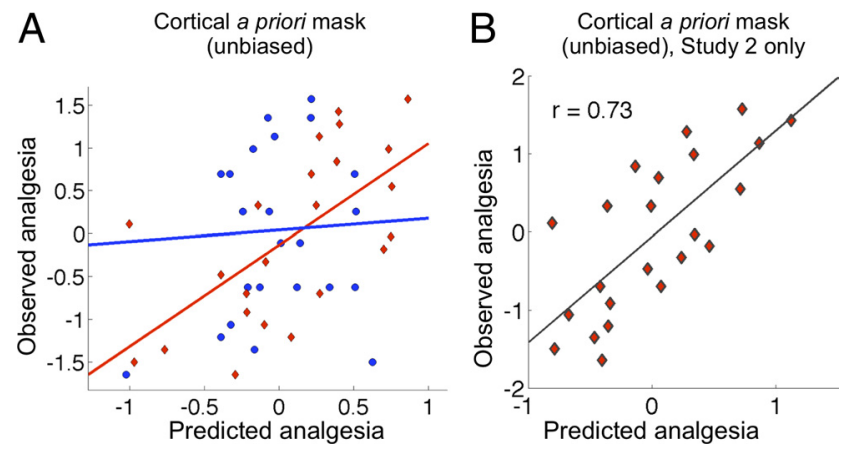

Figure 4. Predictive strength of anticipatory activity: unbiased estimates using LASSO-PCR. $A$, Predictive accuracy obtained from LASSO-PCR within an a priori cortical mask for studies 1 (blue) and 2 (red). The scatter plot shows observed placebo-analgesia scores ( $y$-axis) versus predicted by the cross-validated analyses ( $x$-axis). The accuracy estimate is minimally biased; the cross-validation scheme is known to slightly underestimate predictive accuracy. $\boldsymbol{B}$, Minimally biased predictions from LASSO-PCR for study 2 alone. Predictive accuracy was substantially stronger for study 2 .

that placebo analgesia can be reliably predicted from anticipatory activity in study 2 . We note that the accuracy estimates are conditional on having very little prior estimate of which brain voxels to include in the analysis. These results should help to refine a priori predictions for future studies, and increase prediction accuracy as more precise prior information about the locations of predictive brain regions becomes available.

The LASSO-PCR regression also provided maps of which voxels were important for predicting placebo analgesia, and we compared these maps with the standard regression approach used in analysis 1 . Thresholded LASSO-PCR voxel weights (FDR $q=$ 0.05 corrected, $p<0.016$ ) yielded patterns that were similar to the standard analysis. For comparison with the standard approach, surface renderings of the results are shown in Figure $3 C$ (compare with Fig. $3 A, B$ ). Placebo analgesia was most strongly predicted by placebo-related anticipatory increases in the lateral PFC and superior parietal cortex, pre-SMA, precuneus, and midlateral OFC. Results of a direct comparison between LASSO-PCR 
and standard regression are shown in Figure 5. Figure $5 A$ shows the overlap and disjunction between significant voxels in the LASSO-PCR and standard brain-placebo analgesia correlations. Positive voxel weights are shown in red (LASSO), yellow (standard regression), and orange (overlap), and negative voxel weights are shown in dark blue (LASSO), cyan (standard regression), and medium blue (overlap).

Overlap between the standard regression and LASSO-PCR was observed in virtually every distinct region; however, notably, an area in the pregenual anterior cingulate (pgACC) and nearby rostral medial prefrontal cortex (rmPFC) contributed to the LASSO-PCR pattern, but was not identified with standard regression in analysis 1 . The pgACC/rmPFC finding is meaningful, as pgACC activity during anticipation was correlated with lower expectations of analgesia (Fig. $2 B$ ), and has been shown to positively relate to anticipatory anxiety in other settings (Wager et al., 2009a,b), placebo-induced opioid release (Wager et al., 2007b; Eippert et al., 2009b), and placebo-related increases during pain (Petrovic et al., 2002; Bingel et al., 2006; Eippert et al., 2009b).

A more quantitative comparison between the standard regression and LASSOPCR maps is shown in Figure $5 B$. Voxel weights for LASSO-PCR versus standard regression are plotted for all in-brain voxels (blue points), with some of the key regions from the LASSO-PCR analysis color coded (see figure legend for details). The relationship between the two maps was strongly linear, and regression weights for the LASSO-PCR predictive regions were consistent with the linear relationship across the brain, validating the interpretability of the LASSO-PCR maps, even though the procedure uses many voxels $(\sim 170,000$ in this case) to predict a single outcome variable. Interpretable regression weights are an advantageous feature of some regularized regression techniques (Grosenick et al., 2009) that is not generally shared by many other predictive algorithms.

A plot of the $p$ values for LASSO-PCR versus standard regression, shown in Figure $5 C$, revealed two additional pieces of information. First, low $p$ values (significant voxels) tended to be lower for LASSO-PCR than standard regression (see the figure legend for details). Second, two regions identified in the LASSO-PCR analysis-left OFC and the pgACC/rmPFC—showed much lower $p$ values in the LASSO-PCR regression than standard regression, whereas others (e.g., SII) did not differ in significance across the two analyses. This indicates that these two regions may make a contribution to predictive accuracy that is masked unless the contributions of other brain regions are controlled for.

Analysis 3: comparison of predictive accuracy of anticipatory activity in regions associated with emotional appraisal, cognitive control, and pain The goal of this analysis was to compare predictive accuracy in consensus regions commonly activated by three types of psycho- logical processes (emotional appraisal, cognitive control, and pain processing) that have been implicated in placebo analgesia to gain some insight into which other task types are associated with the most highly predictive regions. The three a priori masks, each derived from a meta-analysis, are shown in Figure $6 \mathrm{~A}$.

The results showed that the emotional appraisal mask was the only one in which anticipatory activity predicted a significant amount of the variance in placebo analgesia. For the executive working memory mask, prediction-outcome correlations were very low and not significant: $r=0.03$ overall $(\mathrm{PE}=1.18, p>0.1$, a $38 \%$ increase in outcome variance relative to the null model, as shown in Fig. $6 B)$ and was low for both studies $(r=-0.13$ and $r=0.19)$. For the emotional appraisal mask, prediction-outcome correlations were significant and comparable to the cortical mask results in analysis $2: r=0.33$ overall $(\mathrm{PE}=0.95, p=0.019$, a $9 \%$ reduction in outcome variance). As with the cortical mask results in analysis 2, predictions were substantially better for study $2(r=0.58$, a $34 \%$ reduction in placebo analgesia error variance) than study 1 ( $r=0.07$, a $10 \%$ increase in error variance). Finally, for pain-related voxels in the PPN mask, prediction-outcome correlations were modest and not significant: $r=$ 
A

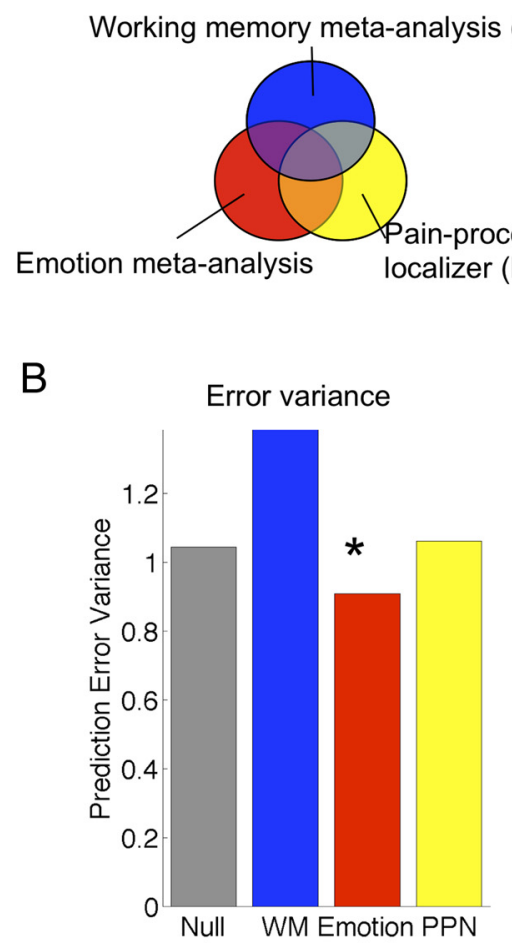

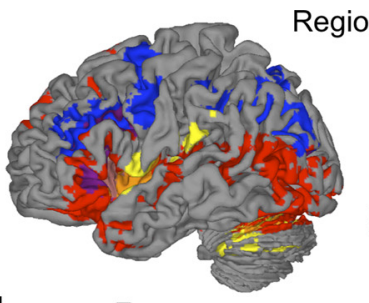

Regions included in each a priori mask
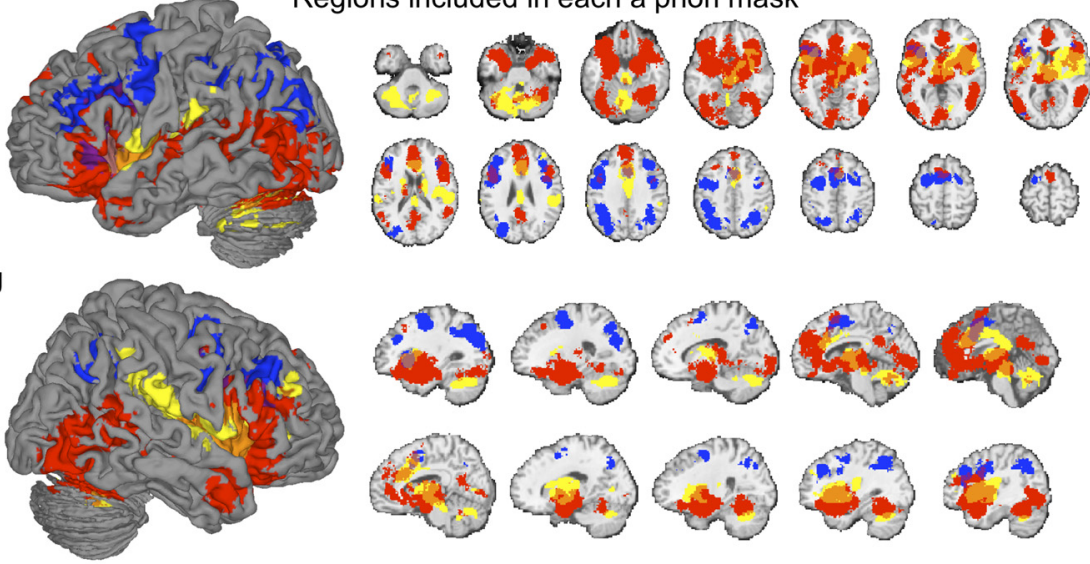

C

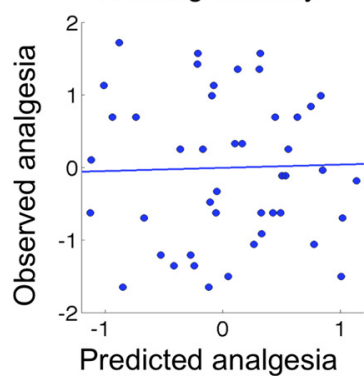

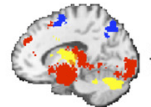
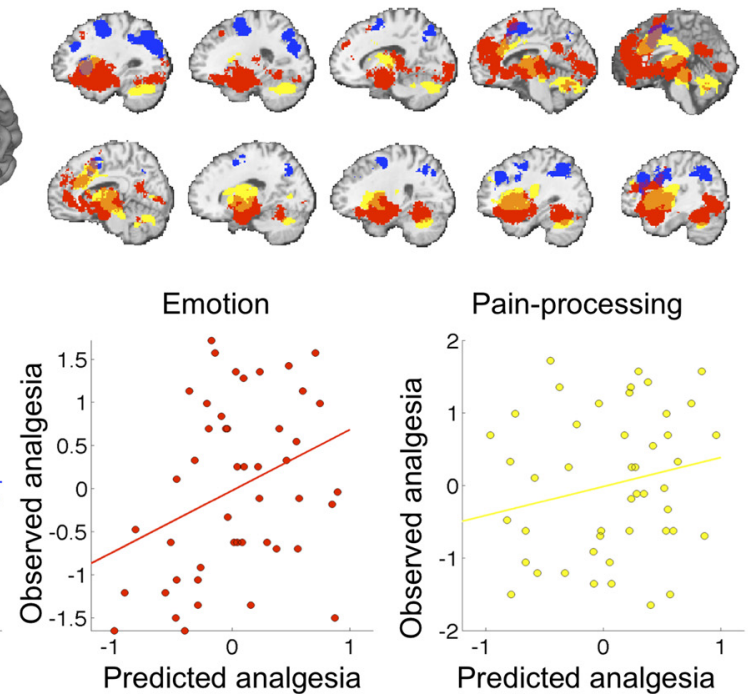

Figure 6. LASSO-PCR based accuracy for predicting placebo analgesia from anticipatory activity within three a priori sets of regions. A, Regions identified in each meta-analysis. Blue, Consistent activations related to executive working memory in a meta-analysis of 60 studies (Wager and Smith, 2003). Red, Consistent activations related to emotional tasks in a meta-analysis of 163 studies (Kober et al., 2008). Yellow, Results from a mega-analysis of 114 participants who underwent a thermal pain challenge in our laboratory (the pain-processing network localizer; see supplemental Fig. 4, available at www.jneurosci.org as supplemental material). $\boldsymbol{B}$, Prediction accuracy from LASSO-PCR for each set of regions, compared with the "null" model based only on training-set placebo analgesia scores. Only the emotion-related regions showed an above-chance $\left({ }^{*} p<0.05\right)$ reduction in prediction error. $C$, Prediction $(x$-axis $)$ - outcome $(y$-axis $)$ scatter plots for each set of regions.

0.22 overall $(\mathrm{PE}=1.03, p>0.1$, a $6 \%$ increase in error variance; $r=0.01$ for study 1 and $r=0.45$ for study 2). Thus, as shown in Figure 6, $B$ (prediction error variance) and $C$ (scatter plots), only the regions from the emotion meta-analysis predicted placebo analgesia. On inspection, the working memory regions are located in more posterior frontal and parietal regions than the results from both standard regression and LASSO-PCR analysis.

\section{Analysis 4: unbiased estimates of the predictive accuracy of anticipatory activity for PPN activity using LASSO-PCR}

To assess how strongly anticipatory $[\mathrm{P}-\mathrm{C}]$ activity predicted placebo effects on brain responses during pain in the PPN, we tested voxels that were localized using an independent dataset $(N=114)$ that compared brain responses to subjectively calibrated high versus low-intensity noxious heat. We used anticipatory activity in the broad a priori cortical mask defined above to predict average $[\mathrm{C}-\mathrm{P}]$ differences in the PPN during pain. Cross-validated accuracy in this map was marginally significant $(p=0.08)$, with moderate prediction-outcome correlations overall ( $r=0.27,4 \%$ reduction in outcome variance) and for each study ( $r=0.39$ for study 1 , and $r=0.34$ for study 2 ), as shown in Figure 7A. Voxels with significant loadings (FDR $q<$ 0.05 corrected) included the left dACC and right pre-SMA, subgenual cingulate, superior temporal, and multiple cerebellar areas (Fig. 7B). The pattern of voxels (i.e., the regression weights), however, was uncorrelated with the pattern of anticipatory activity voxels that predicted placebo analgesia in analysis $2(r=0.03$, n.s.), paralleling a nonsignificant correlation between placebo analgesia scores and $[\mathrm{C}-\mathrm{P}]$ differences in average PPN activity $(r=0.06$, n.s.). The overlap plots in Figure $7 C$ reveal almost no overlapping significant voxels, suggesting that anticipatory predictors of placebo analgesia are qualitatively different from anticipatory predictors of placebo effects on PPN responses. The only overlap was in left IFG, pre-SMA, and left cerebellum. These findings were confirmed by direct examination of the weights for the two analyses, shown in Figure 7D. Regions with large weights for predicting reported analgesia (from analysis 2, $x$-axis) had near-zero weights for predicting placebo effects in PPN activity (analysis 4, y-axis). This plot highlights the findings that placeboinduced PPN reductions were predicted by anticipatory increases (positive weights) in mainly midline structures, whereas the strongest predictors for reported pain included increases mainly in orbitofrontal areas (and decreases in some midline areas). Likewise, PPN effects were predicted by relative anticipatory decreases (negative weights) in DLPFC, whereas placebo analgesia was predicted by increases in a nearby, more ventral part of DLPFC/IFC. Overall, the results suggest that reported analgesia and placebo effects in painrelated responses are quite distinct outcomes.

\section{Analysis 5: predictors of placebo analgesia during peak pain using LASSO-PCR}

To assess how strongly placebo effects in PPN activity during pain experience predicted placebo analgesia, we used the LASSO-PCR algorithm on peak-pain-period activation in voxels identified in the independent PPN localizer. Cross-validated accuracy in this map was significant $(p=0.03)$, as shown in Figure $8 A$, with moderate prediction-outcome correlations overall $(r=0.35,2 \%$ reduction in $\mathrm{PE}$ ) and correlations that appeared to be stronger for study 2 ( $r=0.55,24 \%$ reduction in PE) than study $1(r=0.18$, $16 \%$ inflation of $\mathrm{PE})$. Based on the small reduction in variance, 
A

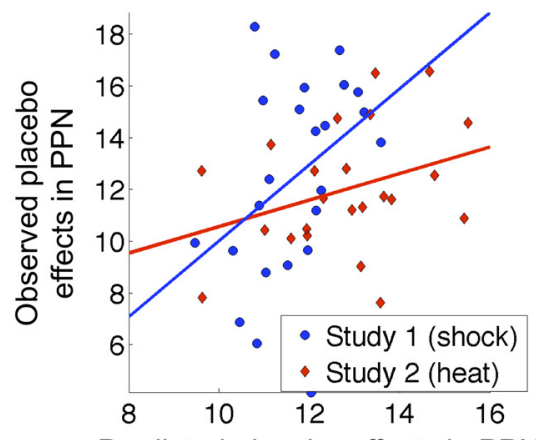

Predicted placebo effects in PPN
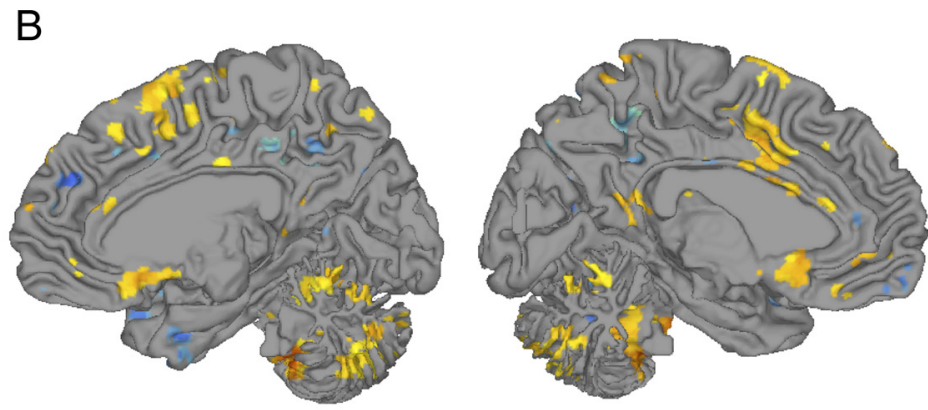

D

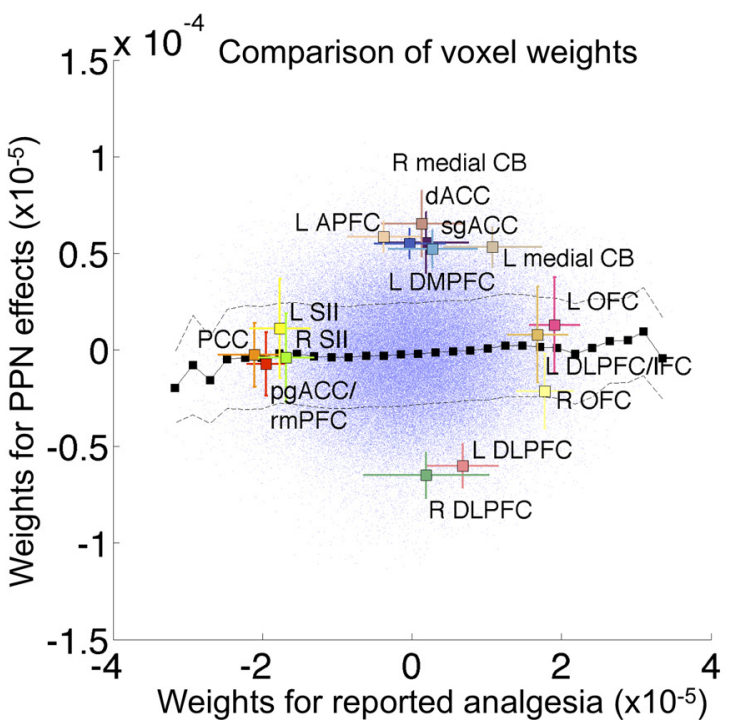

Figure 7. Anticipatory activity predicting placebo effects in average PPN responses from LASSO-PCR (analysis 4). A, Prediction ( $x$-axis) - outcome ( $y$-axis, average [placebo - control] contrast within PPN) scatter plots for each study. B, Map of predictive voxel weights. Colors are as in Figure 3. C, Overlap and dissociations between regions predictive of placebo analgesia in analysis 2 and average placebo effects in PPN (analysis 4). Positive results unique to placebo analgesia are shown in red, those unique to effects in PPN in yellow, and the conjunction in orange. Negative results unique for placebo analgesia are shown in dark blue, unique for effects in PPN in light blue, and the overlap in medium blue. Effects were largely non-overlapping, except in pre-SMA, left inferior frontal gyrus, and cerebellum, suggesting that placebo analgesia and placebo effects in PPN reflect qualitatively distinct anticipatory processes. D, Scatter plot of LASSO-PCR weights predicting reported analgesia ( $x$-axis) versus those predicting PPN effects ( $y$-axis). Colors and lines are as in Figure $5 B$. This plot shows an essentially null relationship between voxel weights for the two outcomes. CB, Cerebellum; APFC, anterior prefrontal cortex; see the text for other abbreviations.

the predictive accuracy is currently not large enough to be of practical use. However, the significant results can still be of value in understanding the relationship between brain activity and placebo analgesia. The voxel weights, shown in Figure $8 B$, reveal that greater placebo analgesia is associated with larger placeboinduced decreases (blue) in anterior cingulate, but also with relative placebo-induced increases (or smaller decreases; yellow) in dorsal anterior and posterior insular cortices. Thus, a simple pattern of reductions throughout pain-processing regions does not appear to characterize large placebo responders.

Interestingly, standard regression predictors of placebo analgesia did not show any significant results in the PPN regions. Rather, strong placebo analgesia was predicted by placeboinduced decreases in several limbic and paralimbic regions thought to play a central role in positive and negative reinforcement value. These regions, shown in supplemental Figure S5 (available at www.jneurosci.org as supplemental material) and listed in supplemental Table S4 (available at www. jneurosci.org as supplemental material), include ventral and dorsal striatum, amygdala, ventral anterior insula, ventromedial PFC, mid-lateral OFC, anterior midbrain, thalamus, posterior cingulate, and the PFC, including the IFG and dorsal MPFC.
To compare the standard regression and LASSO-PCA results, we ran the LASSO-PCA algorithm with a whole-brain mask and compared the results to the standard regression solution (Fig. 8C). Using whole-brain patterns, the prediction was significant overall but not as strong as the PPN-mask results (prediction-outcome $r=0.25, r=0.04$ for study 1 and $r=0.44$ for study $2, p=0.04$, a $3 \%$ reduction in PE). Areas of overlap across standard regression and LASSO-PCR included VMPFC, posterior lateral OFC, striatum, and posterior cingulate. In each region, relative decreases in pain-related responses were associated with greater placebo analgesia. By contrast, in the dorsal anterior insula, relative increases in pain-related responses were associated with greater placebo analgesia (the same results were obtained with the PPN mask). The fact that these patterns contributed highly to predictive accuracy both when LASSO-PCR was conducted within the PPN mask and when it was conducted across the whole brain provides further evidence of the stability of the LASSO-PCR procedure. These findings underscore the general conclusion that large placebo responses are characterized by changes in value-processing circuits rather than a broad inhibition of input to the PPN. 
A

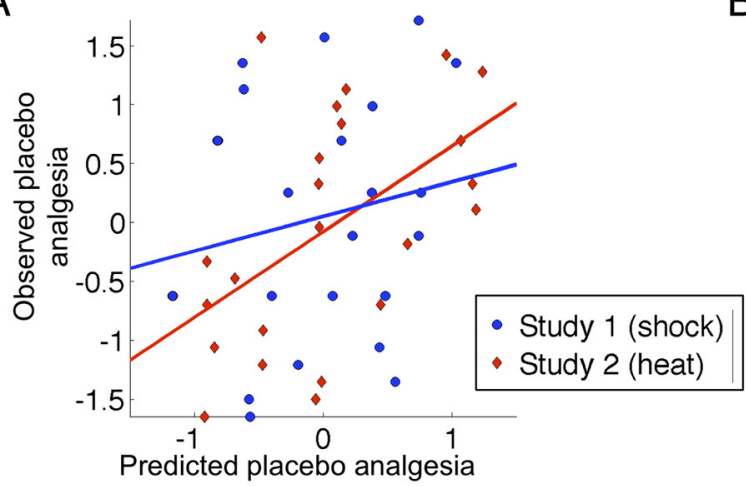

C Comparison between LASSO-PCR weights (whole brain mask) and univariate results
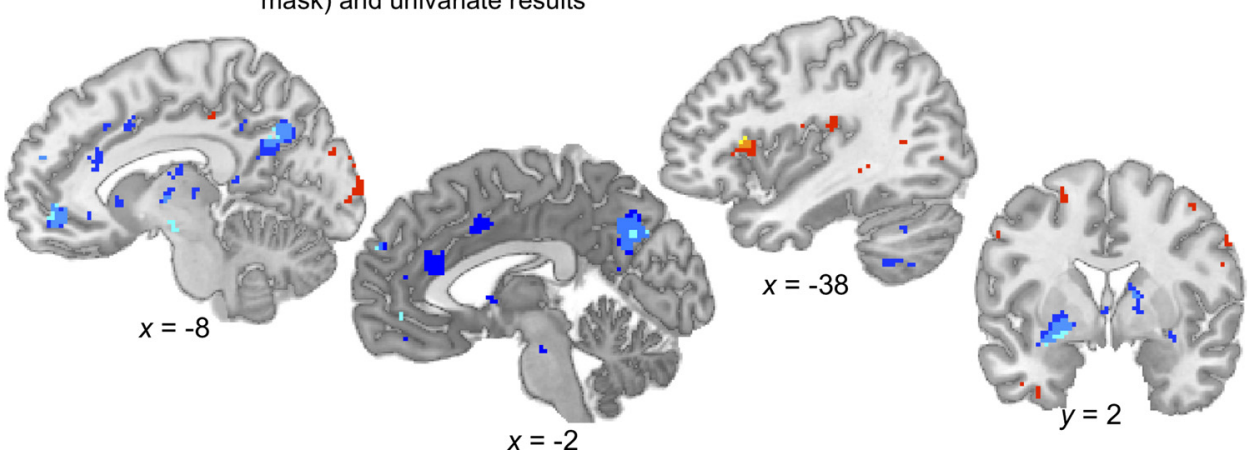

Figure 8. Activity during peak pain (placebo - control) predicting placebo analgesia, from LASSO-PCR (analysis 5). $A$, Prediction ( $x$-axis) versus outcome ( $y$-axis, placebo analgesia) scatter plots for each study. $\boldsymbol{B}$, The left panel shows the PPN mask that constituted the set of predictors, and the right panel shows the most predictive voxel weights ( $p<0.001$ ). Blue indicates areas in which larger placebo-induced decreases during pain predicted placebo analgesia. Yellow/orange indicates areas in which smaller decreases or relative placebo-induced increases predicted placebo analgesia. C, Overlap and dissociations between regions associated with placebo effects in PPN in the LASSO-PCR analysis versus standard regression. Positive results unique to LASSO-PCR are shown in red, those unique to standard regression in yellow, and the conjunction in orange. Negative results unique for LASSO-PCR are shown in dark blue, unique for standard regression in light blue, and the overlap in medium blue. Effects overlapped in posterior cingulate (relative decreases predicted analgesia) and anterior insula (relative increases predicted analgesia), but effects in dorsal cingulate and posterior insula, among other regions, were unique to the LASSO-PCA solution.

\section{Discussion}

Individual variability in placebo responses has been a central issue for many years (Beecher, 1955). Placebo response rates are high in some disorders (Benedetti, 2007) — particularly in pain (Vase et al., 2009), depression (Kirsch et al., 2008; Sneed et al., 2008; Rutherford et al., 2010), and Parkinson's disease (de la FuenteFernandez et al., 2001; Benedetti et al., 2004; Lidstone et al., 2010). Unexplained variability makes it challenging to control placebo responses in clinical trials and capitalize on them in clinical care.

Brain-based measures offer a new way of understanding individual differences in placebo responses (Scott et al., 2007, 2008; Wager et al., 2007b; Eippert et al., 2009a,b; Schweinhardt et al., 2009). However, previous work has been limited because the procedures used were designed to detect nonzero effects, but not to estimate predictive accuracy (Vul et al., 2009; Yarkoni, 2009). Thus, post hoc estimates of brain-placebo correlation strengths are likely inflated, and how accurately patterns of brain activity can predict placebo responses is unknown.

To address this gap, we pooled data from two fMRI studies of placebo analgesia (Wager et al., 2004) $(N=47)$ and used a regularized regression approach (LASSO-PCR) to predict placebo analgesia using all the voxels in an a priori set of regions simultaneously. The technique yields a single test of predictive accuracy integrating across voxels and can be applied to new subjects' data. It also provides interpretable maps across voxels, as suggested by the close correspondence between LASSO-PCR and standard regression voxel weights.

\section{What makes a placebo responder?}

Large placebo analgesia was associated with relative increases during pain anticipation in DLPFC, VLPFC, OFC, superior parietal cortices, precuneus, and lateral cerebellum. Increases in some regions were also correlated with prescan expectations of analgesia. These findings extend earlier reports of anticipatory placebo responses (Wager et al., 2004; Watson et al., 2009; Lu et al., 2010) by showing a direct relationship with individual differences. Conversely, larger placebo analgesia was related to reduced anticipatory responses in SII/temporal regions, possibly reflecting preparatory attentional shifts away from pain (Miron et al., 1989; Quevedo and Coghill, 2007; Buhle and Wager, 2010).

These effects are broadly consistent with changes in several types of mental process, including changes in attention, emotional appraisal, or pain-specific mentation, all of which may cause analgesia (Wiech and Tracey, 2009). However, only consensus regions involved in emotional appraisal [as identified by meta-analysis (Kober et al., 2008)] were found to predict placebo 
analgesia. Surprisingly, regions activated in executive working memory and cognitive control tasks (Wager and Smith, 2003) were not predictive, nor was anticipatory activity in PPN. Thus, anticipatory placebo-related activity seems most strongly associated with affective appraisal processes.

Anticipatory [placebo - control] decreases (or smaller responses) in pgACC/rmPFC also predicted placebo analgesia and were correlated with high prescan expectations of analgesia. The pgACC is a site of placebo-induced opioid release (Zubieta et al., 2005; Wager et al., 2007b; Eippert et al., 2009b). Anticipatory pgACC activity has been associated with anticipatory anxiety (Porro et al., 2003; Wager et al., 2009a), which itself is associated with reduced placebo analgesia (Lyby et al., 2010) and emotional modulation of peripheral responses (Lane and Wager, 2009). Recently, we found that high pain expectancy caused anticipatory increases in a nearby region of medial OFC, which mediated effects on PPN activity (Atlas et al., 2010).

These anticipation-related findings contrast with $\mathrm{pgACC} / \mathrm{rm}$ PFC findings during pain. Several studies show placebo-induced increases during pain (Petrovic et al., 2002; Bingel et al., 2006; Eippert et al., 2009a) and emotional stimulus processing (Petrovic et al., 2005), and relative increases with low pain expectancy that mediate reduced pain report (Atlas et al., 2010). These findings are not necessarily incompatible: Large placebo effects are associated with reduced anticipatory activity in pgACC (and neighboring regions) and larger subsequent increases during pain. However, further research is necessary, as relationships between brain activity and affective valence may be complex. For example, VMPFC connectivity with periaqueductal gray has been associated with both placebo (Bingel et al., 2006; Wager et al., 2007; Eippert et al., 2009b) and anticipatory physiological responses during threat (Wager et al., 2009b).

Perhaps surprisingly, large placebo responses were not characterized by larger decreases in pain responses throughout pain intensity-encoding regions. PPN activity during noxious stimulation did predict a meaningful amount of the variance in placebo analgesia; however, the predictive pattern involved both reductions in cingulate cortex and increases in anterior and dorsal posterior insula. Cingulate decreases are consistent with a central role for the cingulate in pain affect (e.g., Rainville et al., 1997). Dorsal anterior insula increases might be explained in terms of the cognitive/motivational demand of placebo appraisal, as this area responds to a diverse array of tasks involving judgment, appraisal, and cognitive control (Wager and Barrett, 2004; Moulton et al., 2005). However, placebo-related increases in somatosensory-specific posterior insula are harder to explain (Hua et al., 2005). Overall, the complex pattern of predictive pain-related responses may indicate an altered process of pain evaluation in strong placebo responders, rather than a blockade of noxious input [but cf. Matre et al. (2006) and Eippert et al. (2009b)].

Other results also suggest that reported placebo analgesia and placebo effects on noxious stimulus-evoked brain responses are distinct outcomes. These two outcomes were both uncorrelated and associated with different brain regions. Placebo effects on PPN responses were predicted by anticipatory increases in medial structures, including anterior cingulate, ventral cerebellum (supplemental Fig. S6, available at www.jneurosci.org as supplemental material), and subgenual cingulate, paralleling findings of placebo-enhanced functional connectivity between subgenual cingulate and periaqueductal gray (Bingel et al., 2006). Commonalities between predictors of reported analgesia and placebo effects on PPN activity appeared to be pre-SMA and VLPFC, which are both reliably engaged and coactivated with nucleus accumbens during emotion regulation (Wager et al., 2008) as well as involved in internally motivated action (Alexander et al., 2007).

\section{How well can brain activity predict placebo responses?}

Pattern-based prediction using anticipatory activity explained about $12 \%$ of the variance in placebo analgesia for new subjects ( $\sim 40 \%$ for study 2 ). While it appears relatively modest at first glance, we believe this result is quite encouraging, as placebo analgesia is a complex process likely to be determined by multiple personality traits, including optimism (Geers et al., 2005; Morton et al., 2009), suggestibility (De Pascalis et al., 2002), and approach orientation (Schweinhardt et al., 2009) as well as prior treatment experiences (Colloca and Benedetti, 2006) and in-the-moment expectancies (Zubieta et al., 2006). To provide a comparison from another field, Manolio et al. (2009) recently reviewed efforts to predict the incidence of complex diseases using genomewide association studies. Even in the most successful cases, the modal percentage of variance explained was $\sim 5 \%$. In the current study, the predictive accuracy was reduced by the lack of specific prior knowledge: To provide an unbiased estimate, we were forced to specify an a priori mask that included much of the brain (and pay a price in accuracy), but the study results provide more informative priors for future tests.

\section{Limitations and future directions}

This study provides little information on how predictive brain patterns map onto trait-level characteristics [e.g., optimism; (Geers et al., 2005; Morton et al., 2009)]. In addition, the pain intensity ratings used to assess placebo analgesia likely involved both sensory and affective information (see Materials and Methods); thus, the brain patterns that predict placebo analgesia might differ for different individuals, depending on which aspects of pain were judged. Also, it is unknown how these results will generalize to other populations (such as those with chronic pain) and other types of placebo effects, such those arising from long conditioning (Benedetti et al., 2003).

Finally, predictions were substantially more accurate for study 2 than study 1, particularly in analyses of anticipatory responses. Among other differences, study 2 involved a conditioning procedure as part of the placebo manipulation, which introduced a potentially different process (Colloca et al., 2008, 2010) and increased placebo responses overall. It also used a long-duration (20 s) pain stimulus [associated with larger placebo effects (Vase et al., 2009)] and unpredictable anticipatory intervals. In addition, subjects in study 2 were preselected as placebo responders, and thus "nocebo" responders were largely screened out (Scott et al., 2008). Variability related to conditioning, participant characteristics, and other factors should be systematically investigated.

Overall, these results provide more precise anatomical information for use in future studies and a statistical framework for making predictions and improving them systematically as more data becomes available. Thus, they provide a stepping stone to a better understanding of the sources of individual differences in placebo analgesia.

\section{References}

Alexander MP, Stuss DT, Picton T, Shallice T, Gillingham S (2007) Regional frontal injuries cause distinct impairments in cognitive control. Neurology 68:1515-1523.

Amanzio M, Benedetti F (1999) Neuropharmacological dissection of placebo analgesia: expectation-activated opioid systems versus conditioningactivated specific subsystems. J Neurosci 19:484-494. 
Aslaksen PM, Flaten MA (2008) The roles of physiological and subjective stress in the effectiveness of a placebo on experimentally induced pain. Psychosom Med 70:811-818.

Atlas LY, Bolger N, Lindquist MA, Wager TD (2010) Brain mediators of predictive cue effects on perceived pain. J Neurosci 30:12964-12977.

Beecher HK (1955) The powerful placebo. J Am Med Assoc 159:1602-1606.

Benedetti F (2007) Placebo and endogenous mechanisms of analgesia. Handb Exp Pharmacol (177):393-413.

Benedetti F, Pollo A, Lopiano L, Lanotte M, Vighetti S, Rainero I (2003) Conscious expectation and unconscious conditioning in analgesic, motor, and hormonal placebo/nocebo responses. J Neurosci 23:4315-4323.

Benedetti F, Colloca L, Torre E, Lanotte M, Melcarne A, Pesare M, Bergamasco B, Lopiano L (2004) Placebo-responsive Parkinson patients show decreased activity in single neurons of subthalamic nucleus. Nat Neurosci 7:587-588.

Benedetti F, Mayberg HS, Wager TD, Stohler CS, Zubieta JK (2005) Neurobiological mechanisms of the placebo effect. J Neurosci 25:10390-10402.

Berns GS, Chappelow J, Cekic M, Zink CF, Pagnoni G, Martin-Skurski ME (2006) Neurobiological substrates of dread. Science 312:754-758.

Bijur PE, Silver W, Gallagher EJ (2001) Reliability of the visual analog scale for measurement of acute pain. Acad Emerg Med 8:1153-1157.

Bingel U, Lorenz J, Schoell E, Weiller C, Büchel C (2006) Mechanisms of placebo analgesia: rACC recruitment of a subcortical antinociceptive network. Pain 120:8-15.

Bishop CM (2006) Pattern recognition and machine learning. New York: Springer.

Boly M, Balteau E, Schnakers C, Degueldre C, Moonen G, Luxen A, Phillips C, Peigneux P, Maquet P, Laureys S (2007) Baseline brain activity fluctuations predict somatosensory perception in humans. Proc Natl Acad Sci U S A 104:12187-12192.

Brown CA, Seymour B, Boyle Y, El-Deredy W, Jones AK (2008a) Modulation of pain ratings by expectation and uncertainty: behavioral characteristics and anticipatory neural correlates. Pain 135:240-250.

Brown CA, Seymour B, El-Deredy W, Jones AK (2008b) Confidence in beliefs about pain predicts expectancy effects on pain perception and anticipatory processing in right anterior insula. Pain 139:324-332.

Buhle J, Wager TD (2010) Performance-dependent inhibition of pain by an executive working memory task. Pain 149:19-26.

Chapman CR, Casey KL, Dubner R, Foley KM, Gracely RH, Reading AE (1985) Pain measurement: an overview. Pain 22:1-31.

Clark WC, Yang JC, Tsui SL, Ng KF, Bennett Clark S (2002) Unidimensional pain rating scales: a multidimensional affect and pain survey (MAPS) analysis of what they really measure. Pain 98:241-247.

Coghill RC, Sang CN, Maisog JM, Iadarola MJ (1999) Pain intensity processing within the human brain: a bilateral, distributed mechanism. J Neurophysiol 82:1934-1943.

Colloca L, Benedetti F (2006) How prior experience shapes placebo analgesia. Pain 124:126-133.

Colloca L, Tinazzi M, Recchia S, Le Pera D, Fiaschi A, Benedetti F, Valeriani M (2008) Learning potentiates neurophysiological and behavioral placebo analgesic responses. Pain 139:306-314.

Colloca L, Petrovic P, Wager TD, Ingvar M, Benedetti F (2010) How the number of learning trials affects placebo and nocebo. Pain 151:430-439.

de la Fuente-Fernández R, Ruth TJ, Sossi V, Schulzer M, Calne DB, Stoessl AJ (2001) Expectation and dopamine release: mechanism of the placebo effect in Parkinson's disease. Science 293:1164-1166.

De Pascalis V, Chiaradia C, Carotenuto E (2002) The contribution of suggestibility and expectation to placebo analgesia phenomenon in an experimental setting. Pain 96:393-402.

Eippert F, Bingel U, Schoell ED, Yacubian J, Klinger R, Lorenz J, Büchel C (2009a) Activation of the opioidergic descending pain control system underlies placebo analgesia. Neuron 63:533-543.

Eippert F, Finsterbusch J, Bingel U, Büchel C (2009b) Direct evidence for spinal cord involvement in placebo analgesia. Science 326:404.

Geers AL, Helfer SG, Kosbab K, Weiland PE, Landry SJ (2005) Reconsidering the role of personality in placebo effects: dispositional optimism, situational expectations, and the placebo response. J Psychosom Res 58: 121-127.

Genkin A, Lewis DD, Madigan D (2007) Large-scale Bayesian logistic regression for text categorization. Technometrics 49:291-304.

Gracely RH, Dubner R, McGrath PA (1979) Narcotic analgesia: fentanyl reduces the intensity but not the unpleasantness of painful tooth pulp sensations. Science 203:1261-1263.

Grosenick L, Anderson T, Smith SJ (2009) Elastic source selection for in vivo imaging of neuronal ensembles. In: Proceedings of the Sixth IEEE International Symposium on Biomedical Imaging: From Nano to Macro, pp 1263-1266.

Hastie T, Tibshirani R, Friedman J, Franklin J (2005) The elements of statistical learning: data mining, inference and prediction. The Mathematical Intelligencer 27:83-85.

Haxby JV, Gobbini MI, Furey ML, Ishai A, Schouten JL, Pietrini P (2001) Distributed and overlapping representations of faces and objects in ventral temporal cortex. Science 293:2425-2430.

Hua LH, Strigo IA, Baxter LC, Johnson SC, Craig AD (2005) Anteroposterior somatotopy of innocuous cooling activation focus in human dorsal posterior insular cortex. Am J Physiol Regul Integr Comp Physiol 289:R319-R325.

Keltner JR, Furst A, Fan C, Redfern R, Inglis B, Fields HL (2006) Isolating the modulatory effect of expectation on pain transmission: a functional magnetic resonance imaging study. J Neurosci 26:4437-4443.

Kirsch I, Deacon BJ, Huedo-Medina TB, Scoboria A, Moore TJ, Johnson BT (2008) Initial severity and antidepressant benefits: a meta-analysis of data submitted to the Food and Drug Administration. PLoS Med 5:e45.

Kober H, Barrett LF, Joseph J, Bliss-Moreau E, Lindquist K, Wager TD (2008) Functional grouping and cortical-subcortical interactions in emotion: a meta-analysis of neuroimaging studies. Neuroimage 42:998-1031

Kohavi R (1995) A study of cross-validation and bootstrap for accuracy estimation and model selection. In: Proceedings of the Fourteenth International Joint Conference on Artificial Intelligence, pp 1137-1143.

Kong J, Gollub RL, Rosman IS, Webb JM, Vangel MG, Kirsch I, Kaptchuk TJ (2006) Brain activity associated with expectancy-enhanced placebo analgesia as measured by functional magnetic resonance imaging. J Neurosci 26:381-388.

Koyama T, McHaffie JG, Laurienti PJ, Coghill RC (2005) The subjective experience of pain: where expectations become reality. Proc Natl Acad Sci U S A 102:12950-12955.

Kriegeskorte N, Lindquist MA, Nichols TE, Poldrack RA, Vul E (2010) Everything you never wanted to know about circular analysis, but were afraid to ask. J Cereb Blood Flow Metab 30:1551-1557.

Lane RD, Wager TD (2009) The new field of brain-body medicine: what have we learned and where are we headed? Neuroimage 47:1135-1140.

Liberman R (1964) An experimental study of the placebo response under three different situations of pain. J Psychiatr Res 33:233-246.

Lidstone SC, Schulzer M, Dinelle K, Mak E, Sossi V, Ruth TJ, de la FuenteFernández R, Phillips AG, Stoessl AJ (2010) Effects of expectation on placebo-induced dopamine release in Parkinson disease. Arch Gen Psychiatry 67:857-865.

Lieberman MD, Jarcho JM, Berman S, Naliboff BD, Suyenobu BY, Mandelkern M, Mayer EA (2004) The neural correlates of placebo effects: a disruption account. Neuroimage 22:447-455.

Lieberman MD, Berkman ET, Wager TD (2009) Correlations in social neuroscience aren't voodoo: commentary on Vul et al. (2009). Perspect Psychol Sci 4:299-307.

Lorenz J, Hauck M, Paur RC, Nakamura Y, Zimmermann R, Bromm B, Engel AK (2005) Cortical correlates of false expectations during pain intensity judgments - a possible manifestation of placebo/nocebo cognitions. Brain Behav Immun 19:283-295.

Lu H-C, Hsieh J-C, Lu C-L, Niddam DM, Wu Y-T, Yeh T-C, Cheng C-M, Chang F-Y, Lee S-D (2010) Neuronal correlates in the modulation of placebo analgesia in experimentally-induced esophageal pain: a 3T-fMRI study. Pain 148:75-83.

Lyby PS, Aslaksen PM, Flaten MA (2010) Is fear of pain related to placebo analgesia? J Psychosom Res 68:369-377.

Manolio TA, Collins FS, Cox NJ, Goldstein DB, Hindorff LA, Hunter DJ, McCarthy MI, Ramos EM, Cardon LR, Chakravarti A, Cho JH, Guttmacher AE, Kong A, Kruglyak L, Mardis E, Rotimi CN, Slatkin M, Valle D, Whittemore AS, Boehnke M, et al. (2009) Finding the missing heritability of complex diseases. Nature 461:747-753.

Matre D, Casey KL, Knardahl S (2006) Placebo-induced changes in spinal cord pain processing. J Neurosci 26:559-563.

Melzack R, Casey KL (1968) Sensory, motivational, and central control de- 
terminants of pain. In: The skin senses (Kenshalo DR, ed), pp 423-439. Springfield: Thomas.

Michalski RS, Carbonell JG, Mitchell TM (1986) Machine learning: an artificial intelligence approach. San Mateo, CA: Kaufmann.

Miron D, Duncan GH, Bushnell MC (1989) Effects of attention on the intensity and unpleasantness of thermal pain. Pain 39:345-352.

Mitchell TM, Shinkareva SV, Carlson A, Chang K-M, Malave VL, Mason RA, Just MA (2008) Predicting human brain activity associated with the meanings of nouns. Science 320:1191-1195.

Morton DL, Watson A, El-Deredy W, Jones AKP (2009) Reproducibility of placebo analgesia: effect of dispositional optimism. Pain 146:194-198.

Morton DL, El-Deredy W, Watson A, Jones AK (2010) Placebo analgesia as a case of a cognitive style driven by prior expectation. Brain Res 1359:137-141.

Moulton EA, Keaser ML, Gullapalli RP, Greenspan JD (2005) Regional intensive and temporal patterns of functional MRI activation distinguishing noxious and innocuous contact heat. J Neurophysiol 93:2183-2193.

Petrovic P, Kalso E, Petersson KM, Ingvar M (2002) Placebo and opioid analgesia_ imaging a shared neuronal network. Science 295:1737-1740.

Petrovic P, Dietrich T, Fransson P, Andersson J, Carlsson K, Ingvar M (2005) Placebo in emotional processing -induced expectations of anxiety relief activate a generalized modulatory network. Neuron 46:957-969.

Ploner M, Lee MC, Wiech K, Bingel U, Tracey I (2010) Prestimulus functional connectivity determines pain perception in humans. Proc Natl Acad Sci U S A 107:355-360.

Porro CA, Baraldi P, Pagnoni G, Serafini M, Facchin P, Maieron M, Nichelli $P$ (2002) Does anticipation of pain affect cortical nociceptive systems? J Neurosci 22:3206-3214.

Porro CA, Cettolo V, Francescato MP, Baraldi P (2003) Functional activity mapping of the mesial hemispheric wall during anticipation of pain. Neuroimage 19:1738-1747.

Price DD, Barrell JJ, Gracely RH (1980) A psychophysical analysis of experimential factors that selectively influence the affective dimension of pain. Pain 8:137-149.

Price DD, Harkins SW, Baker C (1987) Sensory-affective relationships among different types of clinical and experimental pain. Pain 28:297-307.

Price DD, Craggs J, Verne GN, Perlstein WM, Robinson ME (2007) Placebo analgesia is accompanied by large reductions in pain-related brain activity in irritable bowel syndrome patients. Pain 127:63-72.

Quevedo AS, Coghill RC (2007) Attentional modulation of spatial integration of pain: evidence for dynamic spatial tuning. J Neurosci 27: 11635-11640.

Rainville P, Duncan GH, Price DD, Carrier B, Bushnell MC (1997) Pain affect encoded in human anterior cingulate but not somatosensory cortex. Science 277:968-971.

Rutherford B, Sneed J, Devanand D, Eisenstadt R, Roose S (2010) Antidepressant study design affects patient expectancy: a pilot study. Psychol Med 40:781-788.

Schweinhardt P, Seminowicz DA, Jaeger E, Duncan GH, Bushnell MC (2009) The anatomy of the mesolimbic reward system: a link between personality and the placebo analgesic response. J Neurosci 29:4882-4887.

Scott DJ, Stohler CS, Egnatuk CM, Wang H, Koeppe RA, Zubieta JK (2008) Placebo and nocebo effects are defined by opposite opioid and dopaminergic responses. Arch Gen Psychiatry 65:220-231.

Scott DJ, Stohler CS, Egnatuk CM, Wang H, Koeppe RA, Zubieta JK (2007) Individual differences in reward responding explain placebo-induced expectations and effects. Neuron 55:325-336.

Shao J (1993) Linear model selection by cross-validation. J Am Stat Assoc 88:486-494.
Shapiro AK, Struening EL, Shapiro E (1979) The reliability and validity of a placebo test. J Psychiatr Res 15:253-290.

Sneed JR, Rutherford BR, Rindskopf D, Lane DT, Sackeim HA, Roose SP (2008) Design makes a difference: a meta-analysis of antidepressant response rates in placebo-controlled versus comparator trials in late-life depression. Am J Geriatr Psychiatry 16:65-73.

Tibshirani R (1996) Regression shrinkage and selection via the lasso. J R Stat Soc Series B Stat Methodol 58:267-288.

Vase L, Robinson ME, Verne GN, Price DD (2003) The contributions of suggestion, desire, and expectation to placebo effects in irritable bowel syndrome patients. An empirical investigation. Pain 105:17-25.

Vase L, Petersen GL, Riley JL 3rd, Price DD (2009) Factors contributing to large analgesic effects in placebo mechanism studies conducted between 2002 and 2007. Pain 145:36-44.

Vul E, Harris C, Winkielman P, Pashler H (2009) Puzzlingly high correlations in fMRI studies of emotion, personality, and social cognition. Perspect Psychol Sci 4:274-290.

Wager TD, Smith EE (2003) Neuroimaging studies of working memory: a meta-analysis. Cogn Affect Behav Neurosci 3:255-274.

Wager TD, Rilling JK, Smith EE, Sokolik A, Casey KL, Davidson RJ, Kosslyn SM, Rose RM, Cohen JD (2004) Placebo-induced changes in FMRI in the anticipation and experience of pain. Science 303:1162-1167.

Wager TD, Lindquist M, Kaplan L (2007a) Meta-analysis of functional neuroimaging data: current and future directions. Soc Cogn Affect Neurosci 2:150-158.

Wager TD, Scott DJ, Zubieta JK (2007b) Placebo effects on human $\mu$-opioid activity during pain. Proc Natl Acad Sci U S A 104:11056-11061.

Wager TD, Davidson ML, Hughes BL, Lindquist MA, Ochsner KN (2008) Prefrontal-subcortical pathways mediating successful emotion regulation. Neuron 59:1037-1050.

Wager TD, Waugh CE, Lindquist M, Noll DC, Fredrickson BL, Taylor SF (2009a) Brain mediators of cardiovascular responses to social threat, Part I: Reciprocal dorsal and ventral sub-regions of the medial prefrontal cortex and heart-rate reactivity. Neuroimage 47:821-835.

Wager TD, van Ast VA, Hughes BL, Davidson ML, Lindquist MA, Ochsner KN (2009b) Brain mediators of cardiovascular responses to social threat, part II: Prefrontal-subcortical pathways and relationship with anxiety. Neuroimage 47:836-851.

Watson A, El-Deredy W, Vogt BA, Jones AK (2007) Placebo analgesia is not due to compliance or habituation: EEG and behavioural evidence. Neuroreport 18:771-775.

Watson A, El-Deredy W, Iannetti GD, Lloyd D, Tracey I, Vogt BA, Nadeau V, Jones AK (2009) Placebo conditioning and placebo analgesia modulate a common brain network during pain anticipation and perception. Pain 145:24-30.

Wiech K, Tracey I (2009) The influence of negative emotions on pain: behavioral effects and neural mechanisms. Neuroimage 47:987-994.

Yarkoni T (2009) Big correlations in little studies: inflated FMRI correlations reflect low statistical power-commentary on Vul et al. (2009). Perspect Psychol Sci 4:294-298.

Zeng X, Martinez TR (2000) Distribution-balanced stratified crossvalidation for accuracy estimation. J Exp Theor Artif Intell 12:1-12.

Zubieta J-K, Bueller JA, Jackson LR, Scott DJ, Xu Y, Koeppe RA, Nichols TE, Stohler CS (2005) Placebo effects mediated by endogenous opioid activity on $\mu$-opioid receptors. J Neurosci 25:7754-7762.

Zubieta J-K, Yau W-Y, Scott DJ, Stohler CS (2006) Belief or Need? Accounting for individual variations in the neurochemistry of the placebo effect. Brain Behav Immun 20:15-26. 